Voix et Images

\title{
Portrait de Minerve peint par elle-même, entrevue avec Monique Bosco
}

\section{André Brochu}

Volume 9, numéro 3, printemps 1984

Monique Bosco

URI : https://id.erudit.org/iderudit/200475ar

DOI : https://doi.org/10.7202/200475ar

Aller au sommaire du numéro

Éditeur(s)

Université du Québec à Montréal

ISSN

0318-9201 (imprimé)

1705-933X (numérique)

Découvrir la revue

Citer ce document

Brochu, A. (1984). Portrait de Minerve peint par elle-même, entrevue avec Monique Bosco. Voix et Images, 9(3), 5-12. https://doi.org/10.7202/200475ar d'utilisation que vous pouvez consulter en ligne.

https://apropos.erudit.org/fr/usagers/politique-dutilisation/ 


\title{
Portrait de Minerve peint par elle-même, entrevue avec Monique Bosco
}

\author{
par André Brochu, Université de Montréal
}

V.I. Vos romans ont souvent une allure autobiographique, et il ne faut pas s'y fier car vos héroïnes ont des passés peu ressemblants. Vous êtes vous-même plutôt avare de confidences sur votre vie. Ne pourriez-vous cependant évoquer à grands traits votre passé, les événements et les circonstances qui ont fait de vous ce que vous êtes? Qu'aimeriez-vous qu'on sache sur vous, dans vingt ans ou dans cinquante ans?

M.B. Rien, sans doute, n'est plus angoissant pour moi que de répondre, de façon cohérente, à des questions. Déjà à la petite école j'appréhendais toujours le moment où le sort, ou la maîtresse, me désignerait... Je vais donc, de mon mieux, tenter de répondre à votre question, une triple question, en quelque sorte.

Sans doute, mes romans, surtout le premier, peuvent donner l'impression d'être autobiographiques et il est bien évident que j'y ai mis beaucoup de souvenirs, surtout des années de guerre. Mais aucun n'est réellement «autobiographique», au sens strict. Même si, dans la vie, je suis plutôt bavarde, je répugne à me «livrer» et je suis assez «avare» de confidences. Disons, pour résumer, que je suis née en Autriche, arrivée à Paris à l'âge de 4 ans. J'ai vécu en France jusqu'en 1948, et émigré au Canada. Depuis 1948, je vis donc à Montréal, même si je fais de fréquents séjours en Europe. Bien des choses s'expliquent par ces déracinements et enracinements qui sont toujours, je crois, présents dans ce que j'écris. Dans vingt ou cinquante ans, je suppose que personne ne se souviendra de moi... mais comme c'est la seule forme d' «immortalité» à laquelle on peut aspirer, j'aimerais que l'on puisse dire que j'ai tenté d'être une amie loyale, et un bon prof. 
V.I. Le projet d'écrire vous a-t-il habitée dès votre enfance? Par quelles étapes en êtes-vous arrivée à écrire votre premier livre?

M.B. Très petite, j'ai écrit un roman-mélo, très drôle pour les adultes, qui s'appelait les Deux rivales. J'ai eu un gros succès de public familial. Mais je me suis arrêtée au bout de quelques pages, faute d'inspiration. Et puis rien, pendant des années, sauf quelques poèmes en alexandrins (mais je ne savais pas compter les pieds!... Je ne le sais toujours pas...). Pendant la guerre, je m'étais juré de raconter ce que j'avais éprouvé. J'ai commencé à écrire de façon plus continue quand j'étais étudiante. Mais mon premier roman, il m'a réellement fallu des années pour pouvoir le «mettre en forme» et m'en délivrer.

V.I. Quelle conception vous faites-vous de l'écriture? La voyez-vous surtout liée à l'affirmation de l'individualité, à l'expression de soi, ou comme une aventure du langage (de la forme)?

M.B. Je suis bien incapable de choisir, surtout de réellement analyser ce qui me contraint à écrire. La seule chose qui me vient spontanément à l'esprit, c'est de nier qu'il y ait en moi une conception de l'écriture. Mais je sais la mauvaise foi de toute dénégation trop absolue. Et comme on peut la tourner contre vous. Mais je crois vraiment que je me mets à écrire pour arriver à surmonter un mal de vivre qui serait insoutenable autrement.

V.I. Que pensez-vous de la modernité et de l'inscription de la théorie au coeur de la pratique littéraire?

M.B. Rien. Réellement rien. Quoique, bien sûr, il me paraît évident que nous sommes influencés par la société qui nous entoure et qu'on n'y échappe pas. On est contemporain de tout ce qui se fait et se pense, autour de nous. Et même les plus «classiques» n'échappent pas à la contagion de la mode.

V.I. Que représente pour vous la lecture? Quelle place tient-elle dans votre vie?

M.B. La lecture a toujours occupé une place prédominante dans ma vie. Cela fut, et demeure, une de mes plus fondamentales passions. Même si, comme dans toutes les passions, il y a des «intermittences du coeur', donc des périodes où je ne lis pas - ou peu - ou mal. Des moments de non-vie, où même lire devient impossible. Je préfère les oublier.

V.I. Le fait que vous écriviez a-t-il un impact sur votre façon de lire? Estce que vous lisez pour écrire? 
M.B. Cela serait plutôt le contraire. Quand je suis en train d'écrire, je ne lis rien de "sérieux», rien de beau car je deviendrais habitée par un autre rythme, un autre souffle. Quand je suis en «panne», je préfère me mettre à peindre. Jouer avec les couleurs a souvent sur moi un effet bénéfique qui me permet de passer à la page blanche.

V.I. Quelles lectures et, éventuellement, quels enseignements ont été déterminants pour votre formation intellectuelle et littéraire?

M.B. Toutes les lectures, d'enfance et de jeunesse, faites en vrac, mais avec avidité, voracité, miont nourrie (en une période de restrictions alimentaires justement!). Je ne peux démêler celles qui ont joué un rôle plus important. Quant à la formation «intellectuelle»; j'ai quitté le lycée à l'âge de treize ans et ai seulement suivi des cours à vingt ans, à la Faculté des Lettres de l'U. de M. Là, le Père Ernest Gagnon m'a réellement fait connaître la poésie et les poètes contemporains. Récemment, la rencontre avec Hélène Cixous m'a certainement influencée car j'ai été éblouie a la fois par son oeuvre et son enseignement de la littérature. C'est elle qui m'a fait découvrir Clarice Lispector.

V.I. Vous avez consacré votre thèse de doctorat au thème de l'isolement dans le roman canadien-français, quelques années seulement après votre arrivée au pays, et à une époque où peu de gens s'intéressaient à notre littérature, tant au Québec qu'à l'étranger. Vous êtes une des pionnières des études québécoise modernes. Qu'est-ce qui vous a attirée vers cette littérature? L'isolement que vous avez étudié chez plusieurs romanciers est-il proche parent de celui qui hante vos propres oeuvres? Vous sentez-vous des affinités avec un certain nombre d'écrivains d'ici?

M.B. Quand je suis arrivée à Montréal, je me suis mise à lire, systématiquement, tout ce qui se publiait à l'époque, par curiosité, pour essayer de mieux comprendre ce nouveau pays, ce nouveau continent. Et je ne pensais pas du tout à une thèse. Peu d̀ peu, il m'a semblé qu'on trouvait dans ces oeuvres «quelque chose» à quoi j'ai été sensible et qui me semblait révélateur de la mentalité de l'époque, une sorte de malaise et d'angoisse. Je crois que ce qui m'attirait, ce n'était pas les ressemblances mais au contraire toute l'étrangeté, l'altérité en tout cas. Quant aux affinités avec des écrivains canadiens, j'en ai certainement car plusieurs sont devenues pour moi des amies très proches, précieuses.

V.I. Puisque nous parlons d'affinités culturelles, j'aimerais aborder avec vous la délicate question de ce que vous appelez «l'héritage», dans le poème de Jéricho qui a donné son titre au recueil. Vous écrivez: «Je n'irai jamais à Jéricho. Je renonce à l'héritage. (...) Je reste sage- 
ment à l'abri d'un pauvre présent. Le passé est mort. Et je récuse, d'avance, l'absurde futur incohérent.") Doit-on lire là un rejet de l'identité juive, ou un simple refus du sionisme?

M.B. Ni l'un ni l'autre. Il me semble que, en écrivant ce poème, je me découvrais, et $m$ 'acceptais enfin dans ma solitude essentielle. Non plus penchée vers le passé, ou tournée vers le futur, mais affrontant le présent, au jour le jour, sans aucun éclat de cuivres ou de trompettes.

V.I. Le poème suivant de Jéricho, «L'écarlate carnage», dénonce «l'insatiable soif de sang» chez tous les peuples, même ceux qui sont «demeurés pacifiques, au cours des âges» et qui, maintenant, «sombrent dans une nostalgie anémique». Ce poème vous a-t-il été inspiré par des conjonctures politiques précises?

M.B. Oui, sans doute. Il s'agit d'ailleurs non pas d'un événement mais de toute une série. J'ai été frappée, depuis la fin de la guerre, par cette nostalgie du genre «Ah, que la guerre est jolie». Ce qui fait que toutes les causes, celles des pacifistes, des militants pour les droits et libertés, avaient tant de mal à «recruter» alors qu'il y a toujours des «volontaires» pour les conflits sanglants et armés.

V.I. Vous avez écrit de la poésie, des romans, du théatre radiophonique. Même si vos derniers romans empruntent quelques-unes de leurs techniques au théatre (New Medea) ou à la poésie (Portrait de Zeus peint par Minerve), il s'agit de pratiques de l'écriture différentes, ayant chacune ses exigences, et sans doute aussi ses motivations particulières. Pourriez-vous nous dire ce que chacune représente pour vous?

M.B. Même si je suis prof avant d'être écrivain, j'aurais bien du mal à m'expliquer sur ce qui motive le choix d'une forme, à un moment ou sur un thème donnés. Mais je crois que depuis quelques années j'écris de façon plus ramassée, plus «économique». Comme une sorte d'impatience devant un projet trop long, étiré. Un sentiment d'urgence. Mais surtout un rythme, une respiration qui se fait sans doute plus «courte et haletante» avec l'âge...!

V.I. Vous appréciez beaucoup l'humour, et pourtant votre oeuvre a des accents le plus souvent tragiques, voire désespérés. Un poème, «Bérésina», évoque les «interminables retraites» auxquelles on est condamnée, «trahie de tous», refoulée «toujours plus au nord». Ce schéma est fréquent dans vos romans: les personnages sont peu à peu privés de la possibilité d'un refuge en eux-mêmes, acculés à l'insoutenable, qui a le visage de la mort. Le présent ne fait que reconduire, en les aggravant, les agressions du passé (de l'enfance). Voyez-vous 
votre univers romanesque comme dépespéré? Vous est-il tout à fait impossible d'écrire un livre gai (sinon heureux)?

M.B. Vous avez certainement raison. Il est évident que la vie quotidienne, sans dose d'humour, serait réellement invivable. Et dans la vie, je crois que je ris beaucoup, trop fort peut-être même, mais il me semble que je peux rire, de bon coeur, sans aucune retenue.

Mais de là à écrire un livre qui soit gai, ou surtout heureux, je ne crois pas en être capable. Cela me semblerait une chose bonne et souhaitable mais inatteignable. J'adore les romans de Stendhal, surtout le Rouge et le Noir, et Julien Sorel provoque en moi, par ses réactions, une sorte de sourire, de rire heureux. Mais cela me parait un modèle inimitable.

V.I. On pourrait définir certains de vos romans par un mot qui apparaît dès l'épigraphe de votre premier livre, le mot «lamentation». Le mur des lamentations; Job sur son fumier qui se lamente, ces motifs sont rapportés à la situation de la narratrice. Écrire, c'est faire entendre la voix d'une détresse fondamentale, plus ancienne sans doute que le moi: celle de la créature vouée depuis toujours au malheur. N'y a-t-il pas là un véritable genre littéraire? Je pense à Victor-Lévy Beaulieu qui appelle, précisément, «lamentation» un de ses récits, N'évoque plus que le désenchantement de ta ténèbre, mon si pauvre Abel.

M.B. Je crois qu'il me faut avouer, effectivement, que mon registre se situe plutôt dans la lamentation. C'est un sport que je pratique régulièrement, depuis toujours pour ainsi dire. "Faire le mur», pour moi, cela voulait exactement dire cela: me lamenter, avec abondance, pendant des heures. Et je n'étais jamais à court d'inspiration.

V.I. La narratrice d'Un amour maladroit ne révèle son nom qu'à la fin. Pourtant, dès le début, elle parle de son nom qu'elle présente comme le seul héritage de son père et comme «un nom barbare, peu fait pour être dit, différent des autres». Est-ce le malaise lié à ce nom qui en rend la profération impossible? D'autre part la «femme de Loth", vers le milieu du récit, fait connaître son nom: Hélène. Aveu tardif, comme elle le fait remarquer elle-même. Qu'est-ce qui lui permet d'affirmer son identité, alors que la narratrice d'Un amour maladroit n'y avait pas droit?

M.B. Nommer les choses, les êtres surtout, a longtemps été un tabou. Un tabou presque religieux et que j'ai eu énormément de peine à enfreindre. D'ailleurs, la femme de Loth elle-même n'a qu'un prénom. Pas un nom de famille. C'est la même chose pour Médée, pour Minerve.

V.I. Les Infusoires sont votre seul roman à la troisième personne. Plusieurs histoires y sont menées de front. Quelques employés, hom- 
mes et femmes, d'une agence de publicité se retrouvent en vacances à Venise et tentent, pour une fois, d'échapper aux contraintes quotidiennes et de connaître enfin le bonheur. Qu'est-ce qui vous a attirée, dans ce sujet? La rédaction de ce livre a-t-elle été facile?

M.B. Avec les Infusoires, j'étais consciente d'affronter le problème du deuxième livre. J'ai voulu me prouver que je pouvais «inventer", décrire un milieu, plusieurs personnages. Cela a vraiment été un travail d'apprentissage et $\mathrm{j}$ 'y ai énormément travaillé, même si je considère encore que je n'ai pas réussi, que ce n'est pas au point... Je suis contente de m'être astreinte à ce «labeur».

V.I. La «femme de Loth», conformément au récit biblique, c'est celle qui s'attarde à son passé, lointain et récent; et elle trouve en lui toutes les raisons de désespérer. Mais elle y est pourtant attachée. N'est-ce pas dans ce passé qu'elle trouve ultimement des raisons de vivre? La fin de la Femme de Loth, du reste, est ambiguë: on peut comprendre qu'Hélène va mettre à exécution son projet de suicide - elle n'écrit plus: donc elle est morte - ou qu'elle l'ajourne indéfiniment; n'écrit-elle pas: «La vie reprend sa proie»? Comment voyez-vous le rapport entre présent et passé, dans ce roman et en général?

M.B. Avec la Femme de Loth, je me suis davantage laissée aller, passé et présent confondus, pour essayer de réellement exorciser un désir de destruction, d'auto-destruction. La fin peut paraître ambiguë au lecteur, pour moi elle ne l'est pas - ou plus. J'ai rajouté le dernier paragraphe après des semaines d'hésitations: il me semblait difficile de renoncer à ce suicide sur papier, mais il y a un renoncement au projet initial de mort. Désormais l'héroïne sait, comme la Sonia de Oncle Vania, «qu'il faut supporter» le monde et la vie, et la défaite amoureuse.

V.I. Dans la Femme de Loth, la réciprocité amoureuse est impossible. Hélène n'est jamais aimée dans son corps et dans son âme. Les hommes ne s'attachent jamais qu'à un aspect d'elle-même. Dans ce đivorce entre le corps et l'âme, ou la chair et l'esprit, n'y a-t-il pas un dualisme d'inspiration religieuse - le même qui a longtemps sévi au Québec, et contre lequel s'ingurge un Jean LeMoyne? Aimer Pierre, écrit encore Hélène, c'est «s'engager à lui» comme à Jésus-Christ et pour toujours. L'amour, quand il dépasse le pur attrait sensuel, a-t-il quelque chose qui l'apparente à la religion?

M.B. Pour moi, cela semble une évidence. L'amour, un certain amour, ne peut se vivre que dans une sorte d'intransigeance, d'exigence, un désir d'absolu, d'éternité. C'est ce qui rend la passion si éprouvante, invivable justement. Les lois de l'amour sont aussi inflexibles que les dix commandements. Elles sont sans doute aussi difficiles à respec- 
ter. Mais malheur à qui ne les respecte pas. Une oeuvre comme Paulina 1880 de Pierre-Jean Jouve me paraît exemplaire, à cet égard.

V.I. On peut dire en gros que l'homme, qui est souvent le père ou une figure dérivée du père, est de plus en plus présent dans votre oeuvre. L'enfant d'Un amour maladroit vivait dans un "gynécée» et il faudra du temps avant qu'elle soit mise en présence des hommes. Dans les Infusoires, les hommes sont présents mais restent d'assez pâles figures. Hélène (la Femme de Loth) a un père; et elle vit un véritable amour, au point de vouloir se tuer quand son amant l'abandonne. Dans New Medea, la passion qu'inspire l'homme infidele est encore plus ravageuse. Puis Charles Lévy, m.d. donne la parole à l'homme (au père), pour la première fois si l'on excepte deux courts passages de New Medea; et le père meurt, quand il libère sa fille de son devoir de fidélité à sa race en matière de mariage. Tout tend de plus en plus à se passer entre la fille et le père - Minerve et Zeus; la mère n'est jamais la titulaire du discours, sauf Médée, mais Médée est l'épouse passionnée, l'amante, non la mère. Cette progression vers la confrontation de Minerve et de Zeus a-t-elle le sens d'une remontée vers la scène primitive, la tragédie originaire? Est-ce quelque chose de cet ordre que vous cherchez à circonscrire par l'écriture?

M.B. Je voudrais d'abord dire que dans Charles Lévy, le père ne donne pas vraiment a sa fille le droit de se marier avec qui elle veut. Il se reconnaît, là aussi, impuissant à faire la loi à qui que ce soit. C'est une sorte de démission de sa part. Il est trop malade, trop «mort» déjà, pour même essayer de se battre, de se faire vraiment entendre par elle.

Il me semble que la seule entente indestructible, pour lui du moins, c'est celle qu'il a eue avec sa soeur, sa jumelle. Je crois que la mère, même absente, surtout absente (Médée ne peut se pardonner d'avoir, par sa naissance, tué sa mère) joue un rổle fondamental dans mes «histoires». Plus que le père - même si, ironiquement, on lui concède une toute-puissance au fond imaginaire.

V.I. Dans Charles Lévy, m.d., on observe pour la première fois de nombreux jeux sur le signifiant, à commencer peut-être par ce «m.d.» qui rappelle l'héroïne éponyme du roman précédent. On relève des homophonies partielles ou complètes (Voie des voyages. Voix. Vois."), ou encore, des échos diminués («Un mensonge? Â peine. Un songe plutôt»; «Qu'ai-je compris? Pris? Supporté? Porté avec elle?). À ces procédés, le Portrait de Zeus ajoutera l'agglutination ( $« l l$ doit faire bon-chaud en si nombreuse escorte»; «Dans ma cloque-cloche de verre, il y a moin). Bien entendu, et cela depuis New Medea, la phrase est de plus en plus fragmentée, fragmentale. Quel sens donnez-vous à ce travail sur le signifiant? Est-ce un hasard s'il apparaît en même temps que le thème de la mort du père? 
M.B. Je suis très frappée par votre remarque.

Je crois toutefois que dans ce jeu ou ce «travail sur le signifiant", il y a certainement des influences qui se sont exercées sur moi. Mais aussi, enfant, quand j'étais malade surtout, mais même autrement, je m'amusais - comme tous les enfants sans doute - à désarticuler les mots. J'ai aussi parlé, comme les gens de ma génération, «javanais». C'était idiot et fascinant et servait à faire raler les camarades qui ne connaissaient pas le code. Et si Charles Lévy, à l'hôpital, fait de mauvais jeux de mots sur ses maux, c'est que je crois à un affaiblissement, apporté par la maladie, et à un retour sur soi, celui de la petite enfance. Donc, je crois qu'il s'agit davantage d'une sorte de conjuration contre la mort qui s'avance, que d'une méditation sur le thème de la mort du père. Mais, au fond, là encore, je ne peux, ni ne veux, rien affirmer trop catégoriquement.

V.I. À partir de New Medea, n'y a-t-il pas éviction de la quotidienneté (la grisaille des Infusoires) au profit de la seule tragédie?

M.B. Je serais heureuse d'y avoir réussi.

V.I. Dans votre oeuvre voisinent, et parfois s'entremêlent, des références à la Bible et aux mythologies grecque et romaine. Comment coexistent en vous ces deux traditions culturelles, que beaucoup ont tendance à opposer (de Chateaubriand à Henri Meschonnic)?

M.B. J'ignorais que, pour Chateaubriand, il y avait opposition à entremêler ces références. Cela m'étonne moins de la part de Meschonnic. Quoique, d'une certaine manière, je suppose qu'il y a quelque chose de scandaleux a le faire, surtout quand on se place sur le terrain de l'orthodoxie, d'une orthodoxie ou d'une croyance privilégiée par rapport aux autres. Pour moi, c'est absolument spontané et ne me pose donc aucun problème.

V.I. À quel ouvrage travaillez-vous maintenant?

M.B. À une autre sombre histoire! 